\title{
DEVELOPMENT OF EFFICIENT REAL-FLUID MODEL IN SIMULATING LIQUID ROCKET INJECTOR FLOWS
}

\author{
Gary Cheng* \\ Department of Mechanical Engineering \\ University of Alabama at Birmingham, Birmingham, $\mathrm{AL}$ \\ Richard Farmer ${ }^{\mathrm{H}}$ \\ SECA, Inc., Carson City, NV
}

\begin{abstract}
The characteristics of propellant mixing near the injector have a profound effect on the liquid rocket engine performance. However, the flow features near the injector of liquid rocket engines are extremely complicated, for example supercritical-pressure spray, turbulent mixing, and chemical reactions are present. Previously, a homogeneous spray approach with a real-fluid property model was developed to account for the compressibility and evaporation effects such that thermodynamics properties of a mixture at a wide range of pressures and temperatures can be properly calculated, including liquid-phase, gasphase, two-phase, and dense fluid regions. The developed homogeneous spray model demonstrated a good success in simulating unielement shear coaxial injector spray combustion flows. However, the real-fluid model suffered a computational deficiency when applied to a pressure-based computational fluid dynamics (CFD) code. The deficiency is caused by the pressure and enthalpy being the independent variables in the solution procedure of a pressurebased code, whereas the real-fluid model utilizes density and temperature as independent variables. The objective of the present research work is to improve the computational efficiency of the realfluid property model in computing thermal properties. The proposed approach is called an efficient real-fluid model, and the improvement of computational efficiency is achieved by using a combination of a liquid species and a gaseous species to represent a real-fluid species.
\end{abstract}

* Assistant Professor, Senior Member, AIAA H President, SECA, Inc.
It is found that the overall computational time can be reduced by one order of magnitude by avoiding the stiffness of inverting from the dependent variables (pressure and enthalpy) to independent variables (density and temperature) for multiple-species mixtures. The methodology of the proposed model and thermodynamics properties of some liquid species calculated from the model are reported herein. The accuracy and computational efficiency of the proposed efficient real-fluid model were evaluated by comparing to the numerical result of the developed real-fluid model tested previously.

\section{INTRODUCTION}

With the great demands and competitions for cheaper and safer launchers access to the space, the design of advanced reusable launching vehicles (RLV) is required to not only have higher payload-to-cost ratio, but also improve the reliability and thus extend the life of the reusable launching vehicle. The performance and hardware integrity of liquid rocket engines is greatly influenced by the propellant mixing near the injector. Understanding flow characteristics near the injector is thus very crucial in the advanced liquid rocket engine design. To obtain the information of flow phenomena near the injector, researchers have to rely on either the experimental study or numerical analyses. Conducting hot fire test of liquid injectors not only is too expensive and costly for all preliminary design concepts, but also encounters some technical problems in measuring multi-phase flow properties at the elevated pressure condition. Hence, most of the experimental tests were performed to measure chamber pressure and thrust of the engine. For the 
numerical analysis of the liquid injector, traditionally it has been done by using either 1-D analytical tools, such as $\mathrm{CICM}^{1}$ code, or CFD codes with ideal gas correlation, such as the TDK $\operatorname{code}^{2}$. Though the effect of multiple phases was accounted for in the 1-D analytical toobls, developed based on the cold flow data, the effects of chemical reactions and spatial non-uniformity were not accounted for. Moreover, if the designed operating conditions were out of the range of the database, then the validity of the empirical correction of the analytical code becomes questionable. In addition, the environments of the injector flow of liquid rocket engines are three dimensional because of multiple injectors and the vorticities. Traditionally, the operating conditions of liquid rocket engine are below the critical temperature and well above the critical pressure of propellants. Thus, neither the perfect-gas law is valid, nor the surface tension is stable enough to distinguish the interface between liquid and gaseous phases. Though the CFD methodology has been mature enough to effectively analyze detailed flowfield of combustion, most of CFD codes are using the ideal-gas correlation, and thus the effect of multiple phases is not accounted for. In our previous studies ${ }^{3-6}$, a homogeneous spray approach with a real-fluid property model was developed to simulate the spray combustion problem. The homogeneous spray approach, which assumes equilibrium between liquid and gas phases (i.e. both liquid and gas are treated as continuums, and there are no momentum and heat transfer lags), is suitable for near critical and super-critical spray and can be computationally efficient without tracking liquid droplets because the surface tension of liquid phase either does not exist or is negligible. The real-fluid property model is employed to calculate thermal properties with the inclusion of the compressibility effect and the heat of evaporation.

The homogeneous spray approach with the real-fluid model was incorporated into a welltested pressure-based CFD code, FDNS ${ }^{7-9}$, as a homogeneous spray CFD model to analyze the flowfield near the injector as well as in the chamber and nozzle of the liquid rocket engines. The homogeneous spray CFD model ${ }^{3-6}$ successfully predicted the benchmark test cases of the $2^{\text {nd }}$ International Workshop on Rocket
Combustion Modeling (IWRCM) ${ }^{10-12}$, and other liquid injector configurations ${ }^{13-14}$. However, the developed homogeneous spray CFD model was handicapped by the computational deficiency, especially for the reacting flow. This deficiency is primarily in the iterative matrix inversions for calculating mixture density and temperature from mixture enthalpy and pressure, because the pressure and enthalpy are the independent variables in the CFD solution algorithm, while for the real-fluid mode the density and temperature are the independent variables. The computational deficiency is worsened by the stiffness of the species changes in reacting flows. An efficient real-fluid model is thus proposed to relieve this constrain by representing a real-fluid species with a combination of a gaseous species and a liquid species, where the thermal properties of both species can be modeled by the perfect-gas type of correlations. With this linearization procedure, the enthalpy of the liquid species is a function of temperature only with a known pressure. The matrix inversion process can thus be avoided, and the computational efficiency is greatly improved. The proposed efficient real-fluid model was incorporated into the FDNS code to compute thermal properties of local mixtures with the homogeneous spray approach. The numerical simulation of the RCM test case, previously simulated with the real-fluid model, was repeated with the proposed model to examine its accuracy as well as its computational efficiency. The present study is just a proof of concept that the proposed model can be applied to simulate multiphase spray combustion flows. Any deficiency found from this study will be improved in the future.

\section{NUMERICAL METHODOLOGY}

The characteristics of spray combustion flows are multiple phases and chemical reactions. In order to properly account for the effect of phase change in spray combustion flows, a generalized equation of state (EOS) is required in order to accurately compute the thermal properties (such as density, enthalpy, speed of sound, and etc.) of the liquid propellant over a wide range of operating conditions. A typical pressure-density correlation for a given species can be plotted as shown in 
Figure 1, which consists of liquid, gas, two-phase, and dense fluid regimes. In our previous studies ${ }^{3-}$ 6, a real-fluid model was developed and successfully predicted the compressibility effect of oxygen and nitrogen at an elevated pressure. In the developed real-fluid model, thermal and caloric equations of state, vapor pressure, heat of vaporization, surface tension, and transport properties are modeled with the equations of state proposed by Hirshfelder, et al $^{15-16}$ (we term these the HBMS equations of state) and with conventional correlations ${ }^{17}$, for the other properties. These correlations are explicit in density and temperature, and can be expressed as follows.

HBMS thermal equation of state:

$\frac{P}{P_{c}}=\sum_{j=1}^{4} T_{r}^{j-2} \sum_{i=1}^{6} B_{i j} \rho_{r}^{i-2} ; T_{r} \equiv \frac{T}{T_{c}} \quad ; \quad \rho_{r} \equiv \frac{\rho}{\rho_{c}}$

HBMS caloric equation of state:

$$
\begin{aligned}
\frac{H-H_{0}}{R T}= & Z_{c} \int_{o}^{\rho_{r}}\left[\frac{P}{T_{r}}-\left(\frac{\partial P}{\partial T_{r}}\right)_{\rho_{r}}\right] \rho_{r}^{-2} d \rho_{r} \\
& +Z_{c} \frac{P}{\rho_{r} T_{r}}-1
\end{aligned}
$$

where $\rho_{c}, T_{c}$, and $P_{c}$ are the density, temperature, and pressure at the critical condition, while $\rho_{r}$ and $T_{r}$ are the reduced density and temperature which are normalized values with respect to the value at the critical condition. $H$ and $H_{0}$ are the real-fluid enthalpy and ideal gas enthalpy of a give species. $Z_{c}$ is the compressibility for a given species at the critical point. $B_{i j}$ and $R$ are the coefficients of the thermal property polynomial and the gas constant for a given species, respectively. However, for most of the multiple-species flow solvers either pressure and enthalpy are the primitive variables (pressure based), or the density and enthalpy are the primitive variables (density based). The inversion process from the dependent variables to the independent variables is very computationally intensive, especially for the chemically reacting flows where a mixture consists of multiple species and species concentrations of the mixture can change drastically (stiffness problem). The efficient real-fluid model is proposed to represent a real-fluid species $(S)$ with a mixture of a liquid $\left(S_{l}\right)$ and a gaseous $\left(S_{g}\right)$ species, and can be expressed as

$$
S=(1-f) S_{l}+f S_{g}
$$

where $f$ is the fraction of the gaseous species, and the split between $S_{l}$ and $S_{g}$ is defined by

$$
\rho=(1-f) \rho_{l}+f \rho_{g}
$$

The density $(\rho)$ of the real-fluid species is obtained from the developed real-fluid model. One of the main features of the proposed efficient real-fluid model is that the density-temperature-pressure correlations of both the liquid and gaseous species are similar to the perfect-gas law such that the computational efficiency can be improved. Thus, the density of liquid $\left(\rho_{l}\right)$ and gaseous $\left(\rho_{g}\right)$ species can be explicitly calculated from pressures and temperatures. In addition, for the liquid species a compressibility factor $\left(Z_{c}\right)$, which is a function of temperature, and a fictitious molecular weight $\left(M_{w, l}\right)$ are employed to account for the compressibility effect. With a known pressure, the fictitious molecular weight, the compressibility factor, and the liquid density can be calculated as

$$
M_{w, l}=\frac{\rho_{o} R_{u} T_{0}}{p}
$$

where $\quad \begin{cases}T_{o}=T_{c}, & \rho_{o}=\rho_{c} \text { if } P \geq P_{c} \\ T_{o}=T_{s}, & \rho_{o}=\rho_{s, L} \text { if } P<P_{c}\end{cases}$

$$
\rho_{l}(T)=\frac{P M_{w, l}}{R_{u} Z_{c} T} \quad Z_{c}(T)=\frac{\rho(T)}{\rho_{o}}
$$

$R_{u}, T_{s}$, and $\rho_{s . L}$ are the universal gas constant, saturation temperature, and the saturation liquid density, respectively. The correlation of specific enthalpy between real-fluid species $(h)$, liquid species $\left(h_{l}\right)$, and gaseous species $\left(h_{g}\right)$ can also be expressed as

$$
h=(1-f) h_{l}+f h_{g}
$$


The second main feature of the present model is that for a given pressure the specific heat $\left(C_{p, l}\right)$ and the enthalpy $\left(H_{l}\right)$ of the liquid species are polynomial functions of the temperature only similar to those of the gaseous species of the CEC code $^{18}$, which can be shown as

$$
\frac{C_{p, l}}{R}=\sum_{n=1}^{5} A_{n} T^{n-1} \quad ; \quad \frac{H_{l}}{R}=A_{6}+\sum_{n=1}^{5} \frac{A_{n}}{n} T^{n}
$$

$A_{n}$ are polynomial coefficients of the liquid species, and can be obtained by curve fitting the real-fluid enthalpy for the temperature below either the saturation temperature (at subcritical pressure) or the critical temperature (at supercritical pressure). As a result, the effect of the heat of evaporation will be accounted for by increasing the fraction of gaseous species as the temperature increase. The last main feature of the present model is generating a look-up table for the speed of sound and the ratio of the specific heat based on the developed real-fluid model. The need for the speed of sound and the ratio of specific heat is to satisfy the correlation between the density and the pressure changes, which can be shown as

$$
\Delta \rho= \begin{cases}\Delta P\left(\frac{\partial \rho}{\partial P}\right)_{T} & =\Delta P \frac{\gamma}{a^{2}} \text { :both liquid and gas } \\ \frac{\Delta P M_{w}}{R_{u} T} & : \text { ideal gas only }\end{cases}
$$

This correlation can be applied to both densitybased and pressure-based flow solvers, and is valid for liquid phase, gas phase, two-phase, and dense fluid regions. In order to apply the proposed effective real-fluid model to a flow solver, a preprocessor was constructed to generate the thermal properties of the liquid species for a given pressure. In the present model, the temperature is an explicit function of the specific enthalpy at a given pressure, and the fraction of the gaseous species and the density can be directly calculated based on the temperature as well. As a result, the computational time can be greatly reduced and the time marching step size can be increased because the matrix inversion for calculating mixture density and temperature from mixture enthalpy and pressure is avoided.

The proposed efficient real-fluid model was incorporated into the homogeneous spray methodology in the FDNS ${ }^{7.9}$ code to simulate the spray combustion problem. The framework of the FDNS code is an elliptic, finite difference NavierStokes flow solver, which employs a predictor plus multi-corrector pressure-based solution algorithm. Higher order upwind, total variation diminishing (TVD), or central difference schemes plus adaptive second-order and fourth-order dissipation terms are used to approximate the convection terms of the transport equations. Various matrix solvers, such as vectorized point implicit, conjugate gradient, and generalized minimal residual ${ }^{19}$ (GMRES), are provided in the code such that users can select one for a given transport equation. Since the FDNS flow solver is a structured code, multi-block, multi-zone grid topology was employed in the code so that problems with complex geometries can be analyzed efficiently. Since the flow solver is a pressure-based code, a pressure correction $\left(P^{\prime}\right)$ equation is derived from the continuity equation and can be expressed as:

$$
\begin{aligned}
& \frac{\beta_{p} P^{\prime}}{\Delta t}+\nabla \cdot\left(V_{i}^{*} \beta_{p} P^{\prime}\right)-\nabla \cdot\left(\rho^{*} \mathrm{D}_{p} \nabla P^{\prime}\right) \\
& \quad=-\nabla \cdot\left(\rho^{*} V_{i}^{*}\right)-\frac{\rho^{*}-\rho^{n}}{\Delta t} \\
& P^{n+1}=P^{n}+P^{\prime} ; \quad \beta_{p}=\gamma / a^{2} ; \quad V_{i}^{\prime} \approx-\mathrm{D}_{p} \nabla P^{\prime}
\end{aligned}
$$

where the superscripts * and $n$ denote the value at the intermediate and previous time steps, respectively. $D_{p}$ is the inverse of the matrix of the coefficients of the convective terms in the finitedifference form of the inviscid equations of motion. For the homogeneous spray approach, the speed of sound $(a)$, and the ratio of specific heat $(\gamma)$ of a multi-component mixture can be calculated from the real-fluid properties of its constituents based on the additive volume method, 


$$
\gamma=\frac{\sum_{i} n_{i}\left(C_{p} / R\right)_{i}}{\sum_{i} n_{i}\left(C_{p} / R\right)_{i} / \gamma_{i}} \quad ; \quad a^{2}=\frac{\gamma / M_{w}^{2}}{\sum_{i} \gamma_{i} \alpha_{i} / a_{i}^{2} M_{w, i}^{2}}
$$

where $n_{i}, \alpha_{i}, \gamma_{i}, R_{i},\left(C_{p}\right)_{i}, a_{i}$, and $M_{w, i}$ are the mole fraction, mass fraction, ratio of specific heat, gas constant, specific heat, speed of sound and the molecular weight of species “i”, respectively.

\section{NUMERICAL RESULTS}

In order to utilize the proposed effective realfluid model, thermal properties of the liquid species as well as the split between gaseous and liquid species have to be generated beforehand. A pre-processor was constructed to generate those data needed by the present model based on the real-fluid model. The thermal properties of oxygen, hydrogen and RP-1 generated by the preprocessor are shown in Figures 2-13, respectively. It can be clearly seen that the thermal properties generated by the developed pre-processor accounts for the compressibility and heat of evaporation effects for these three species properly.

To examine the accuracy of the present model, a LOX/GH2 uni-element shear coaxial injector, RCM-3 test case (Ref. 12), was simulated. Previously, the homogeneous real-fluid model was employed to simulate this test case, and good success was reported in Ref. 3 and 5. Thus, the accuracy and computational efficiency of the present model with the homogeneous spray approach can be evaluated comparing to the numerical result of the developed real-fluid model. As illustrated in Table 1 , this is a super-critical spray combustion flow test case. Since surface tension is either zero or not well defined for supercritical conditions, drops either do not exist or are very unstable and do not survive very long. Therefore, the homogeneous spray model is an appropriate and effective approach for simulating this test case. The injector configuration and flow conditions for the RCM-3 test case are presented in Figure 14. A two-zone mesh system $(61 \times 39$ and $301 \times 101$ ) was used to model the injector section and the combustion chamber.
As shown in our previous study ${ }^{3 \& 5}$, the numerical results of the equilibrium chemistry are almost identical to those of the finite-rate chemistry model because the reaction rate of the $\mathrm{H}_{2} / \mathrm{O}_{2}$ combustion at the elevated pressure is extremely fast. Moreover, the focus of the present study is to verify the accuracy of the proposed effective real-fluid model in predicting the thermodynamics properties over a wide range of temperatures. Therefore, it is more computationally efficient to utilize the equilibrium chemistry to simulate the super-critical $\mathrm{O}_{2} / \mathrm{H}_{2}$ combustion test case in the present study. The contours of predicted $\mathrm{OH}$ radical concentrations of both the present and the real-fluid models are shown in Figure 15. It is obvious the numerical results of both models are almost identical. In Figure 16, the oxygen concentration of the realfluid model compares to the concentration of gaseous and liquid oxygen predicted by the present model. The sum of liquid and gaseous oxygen of the present model agrees very well with the oxygen concentration of the real-fluid model.

The radial temperature profiles predicted at several axial stations are shown in Figures 17-19, while the axial temperature profiles at several radial locations are shown in Figures 20-22. The agreement between the present model and the realfluid model is excellent despite some small discrepancy at the location very close to the injector. This minor discrepancy is caused by the approximation that for the entire flow field all the property correlations of the present model are obtained based on a given pressure (chamber pressure in this case), whereas the pressure near the injector deviates substantially from the chamber pressure. This deficiency can be eliminated if the thermal properties are interpolated from property correlations of various pressures based on the local pressure. The thermal property interpolation for the proposed effective real-fluid model is being developed as part of this on-going research project. Nevertheless, for those multi-phase flow problems, where the pressures of the entire flow field do not differ too much from the value that the thermal properties for the liquid species were generated for, the fixed-pressure property approach is very efficient and effective. In addition to the model accuracy, more importantly the overall computational time is 
reduced by one order of magnitude because the numerical simulation of the present model can be conducted with larger time marching step size (from $0.1 \mu \mathrm{sec}$ to $1 \mu \mathrm{sec}$ ). This proves that the present model is able to relieve the stiffness problem by the utilization of two species representing a real-fluid species, as well as decoupling the process of computing the density and temperature.

\section{CONCLUSIONS}

A property pre-processor based on the present model was constructed to generate thermal properties for various liquid species. As can be seen from the generated thermal properties, the effects of compressibility and the heat of evaporation are taken into account by the developed property pre-processor. The present model does meet the goal of improving the computational efficiency of the real-fluid model. Meanwhile, the numerical result of the present model is almost identical to that of the real-fluid model. A minor discrepancy between the present model and the real-fluid model occurs near the injector, which is caused by the pressure deviation from the condition, which the thermal properties of the liquid species were generated for. This deficiency can be overcome by interpolating local thermal properties from a pre-generated property correlations based on the local pressure. The present study is just a proof of concept that the proposed model can be applied to simulate multiphase spray combustion flows. The variablepressure version of the efficient real-fluid model is being developed as part of this on-going research activity. The result of this development will be reported in the future.

\section{ACKNOWLEDGEMENTS}

The authors would like to express their appreciations to Mr. Kevin Tucker and Dr. Jeff West for their valuable advices and supports. This work was performed under NAS8-02062 for the Marshall Space Flight Center of the National Aeronautics and Space Administration.

\section{REFERENCES}

1. Sutton, R.B., Schuman, M.D., and Chadwick, W.D. "Operating Manual for Coaxial Injection Combustion Model," NASA CR-129031, Rocketdyne Division, Rockwell International, Canoga Park, CA, 1 April 1974.

2. Nickerson, G.R., et al, "Two-Dimensional Kinetics (TDK) Nozzle Performance Computer Program," Vols. I-III, Rpt. No. SN91, Software and Engineering Associates, Inc., mar. 1989.

3. Cheng, G.C., and Farmer, R.C., "CFD Spray Combustion Model for Liquid Rocket Engine Injector Analyses," AIAA Paper 2002-0785, 2002.

4. Farmer, R.C., Cheng, G.C., and Chen, Y.S., "CFD simulation of Liquid Rocket Engine Injectors: Test case RCM 1," Proceeding $2^{\text {nd }}$ International Workshop on Rocket Combustion Modeling, Lampoldshausen, Germany, March 25-27, 2001.

5. Farmer, R.C., Cheng, G.C., and Chen, Y.S., "CFD simulation of Liquid Rocket Engine Injectors: Test case RCM 2," Proceeding $2^{\text {nd }}$ International Workshop on Rocket Combustion Modeling, Lampoldshausen, Germany, March 25-27, 2001.

6. Farmer, R.C., Cheng, G.C., and Chen, Y.S., "CFD simulation of Liquid Rocket Engine Injectors: Test case RCM 3," Proceeding $2^{\text {nd }}$ International Workshop on Rocket Combustion Modeling, Lampoldshausen, Germany, March 25-27, 2001.

7. Chen, Y.S., "Compressible and Incompressible Flow Computations with a Pressure Based Method," AIAA Paper 89-0286, 1989.

8. Cheng, G.C., Farmer, R.C., and Chen, Y.S., "Numerical Study of Turbulent Flows with Compressibility Effects and Chemical Reactions," AIAA Paper 94-2026, 1994.

9. Cheng, G.C., Chen, Y.S., and Wang, T.S., "Flow Distribution Within the SSME Main 
Injector Assembly Using Porosity Formulation," AIAA Paper 95-0350, 1995.

10. Telaar, J., Schneider, G., Hussong, J., and Mayer, W., "Cryogenic Jet Injection: Description of Test Case RCM 1," Proceeding $2^{\text {nd }}$ International Workshop on Rocket Combustion Modeling, Lampoldshausen, Germany, March 25-27, 2001.

11. Vingert, L., and Habiballah, M., "Test Case RCM 2: Cryogenic Spray Combustion at 10 bar at Mascotte," Proceeding $2^{\text {nd }}$ International Workshop on Rocket Combustion Modeling, Lampoldshausen, Germany, March 25-27, 2001.

12. Thomas, J.L., et al, "Test Case RCM 3: Supercritical Spray Combustion at 60 bar at Mascotte," Proceeding $2^{\text {nd }}$ International Workshop on Rocket Combustion Modeling, Lampoldshausen, Germany, March 25-27, 2001 .

13. Cheng, G.C., Anderson, P.G., and Farmer, R.C., "Development of CFD Model for Simulating Gas/Liquid Injectors in Rocket Engine Design," AIAA Paper 97-3228, 1997.

14. Farmer, R.C., Cheng, G.C.; Trinh, H., and Tucker, K., "A design Tool for Liquid Rocket Engine Injectors," AIAA 2000-3499, 2000.

15. Hirschfelder, J.O,, et al, "Generalized Equations of State for Gases and Liquids," IEC, 50, pp.375-385, 1958.

16. Hirschfelder, J.O., et al, "Generalized Excess Functions for Gases and Liquids," IEC, $\underline{50}$, pp.386-390, 1958.

17. Reid, R.C., et al, The Properties of Gases \& Liquids, 4th ed., McGraw-Hill, 1987.

18. Gordon, S., and B.J. McBride, "Computer Program for Calculation of Complex Chemical Equilibrium Compositions, Rocket Performance, Incident and Reflected Shocks, and Chapman-Jouget Detonations," NASA-SP$273,1971$.
19. Saad, Y., and Schultz, M.H., SIAM Journal of Sci. Stat. Comput., Vol. 7, pp. 856-869, 1986.

Table 1. RCM-3 Test Case Operating Conditions

\begin{tabular}{|l|c|c|}
\hline Conditions & $\mathrm{H}_{2}$ & $\mathrm{O}_{2}$ \\
\hline Pressure & $6 \mathrm{MPa}$ & $6 \mathrm{MPa}$ \\
\hline Mass flow rate & $70 \mathrm{~g} / \mathrm{s}$ & $100 \mathrm{~g} / \mathrm{s}$ \\
\hline Temperature & $287 \mathrm{~K}$ & $85 \mathrm{~K}$ \\
\hline Density & $5.51 \mathrm{~kg} / \mathrm{m} 3$ & $1177.8 \mathrm{~kg} / \mathrm{m}^{3}$ \\
\hline Cp & $15100 \mathrm{~J} / \mathrm{kg} / \mathrm{K}$ & $1660.9 \mathrm{~J} / \mathrm{kg} / \mathrm{K}$ \\
\hline Velocity & $236 \mathrm{~m} / \mathrm{s}$ & $4.35 \mathrm{~m} / \mathrm{s}$ \\
\hline Viscosity & $8.67 \mathrm{E}-6 \mathrm{~kg} / \mathrm{m} / \mathrm{s}$ & $12.34 \mathrm{E}-4 \mathrm{~kg} / \mathrm{m} / \mathrm{s}$ \\
\hline
\end{tabular}




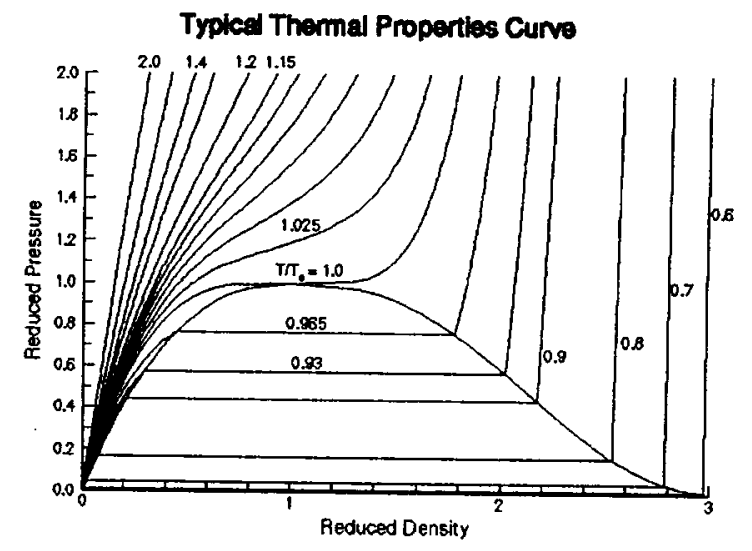

Figure 1. Typical $P-\rho-T$ correlation for a given species

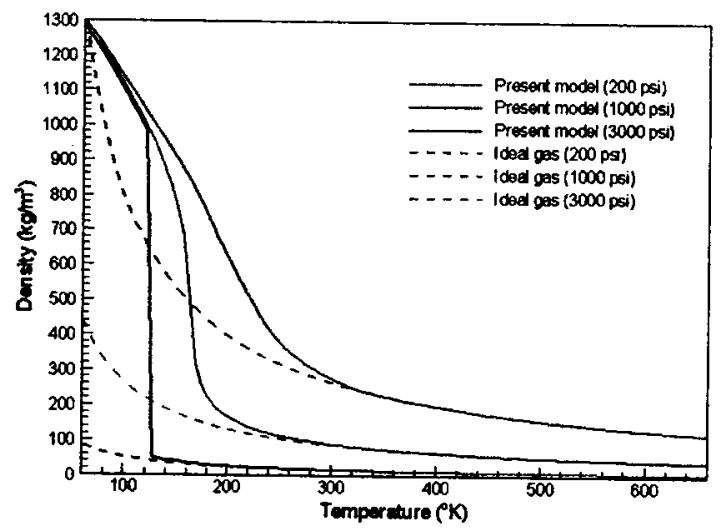

Figure 2. Comparison of oxygen density at various temperatures and pressures

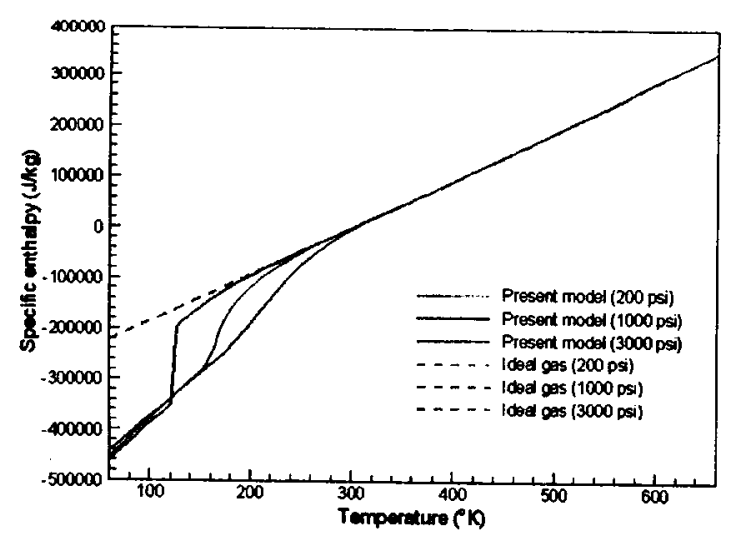

Figure 3. Comparison of oxygen specific enthalpy at various temperatures and pressures

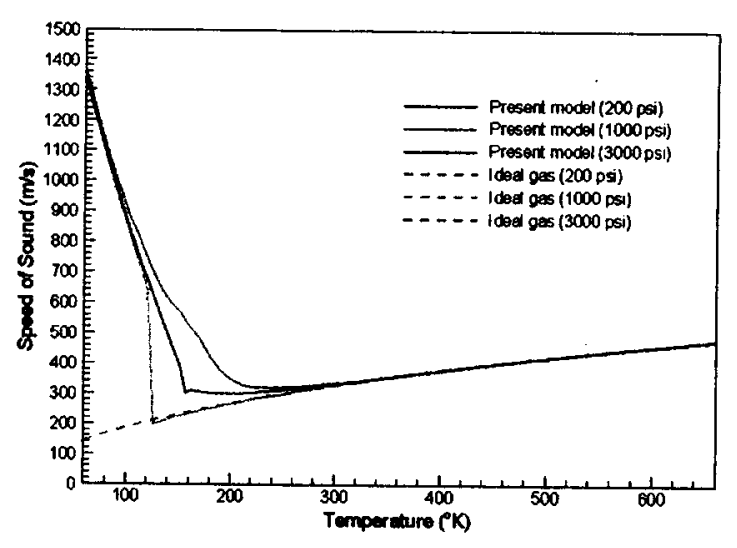

Figure 4. Comparison of oxygen speed of sound at various temperatures and pressures

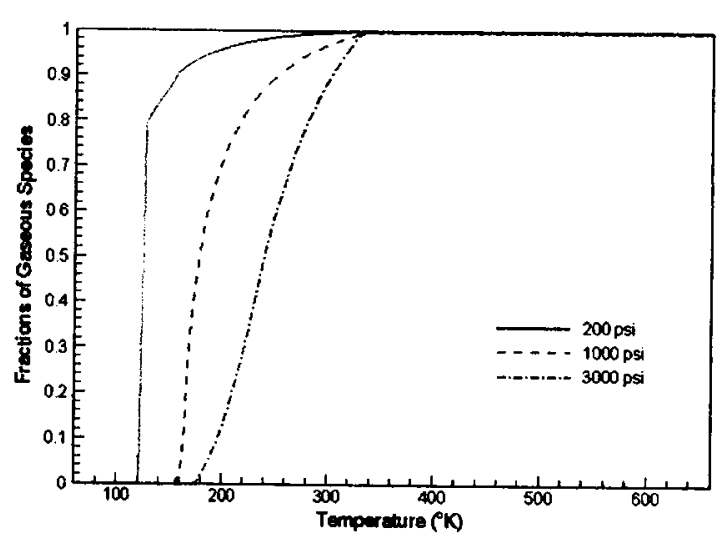

Figure 5. Fractions of gaseous oxygen at various temperatures and pressures

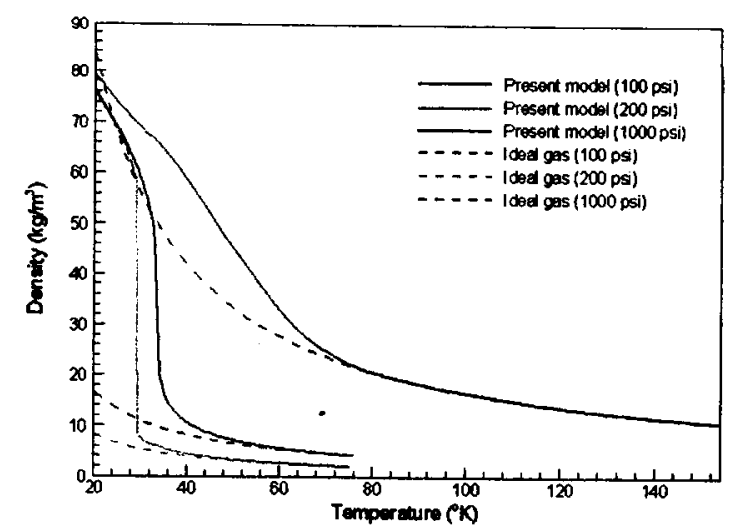

Figure 6. Comparison of hydrogen density at various temperatures and pressures 


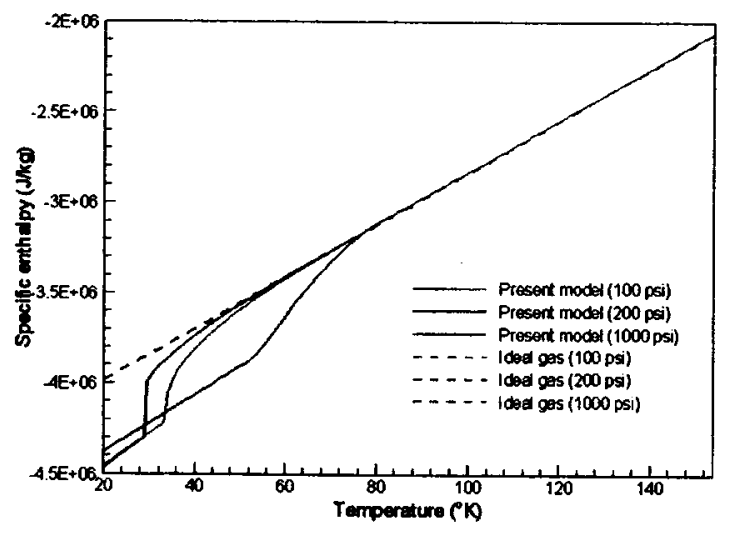

Figure 7. Comparison of hydrogen specific enthalpy at various temperatures and pressures

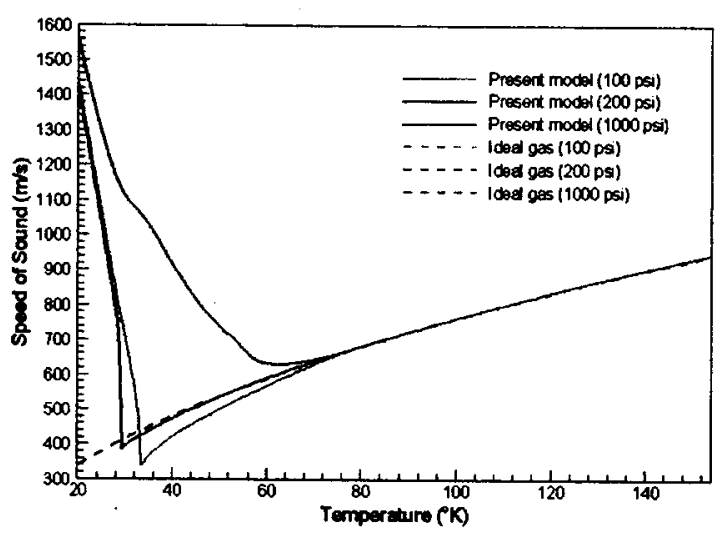

Figure 8. Comparison of hydrogen speed of sound at various temperatures and pressures

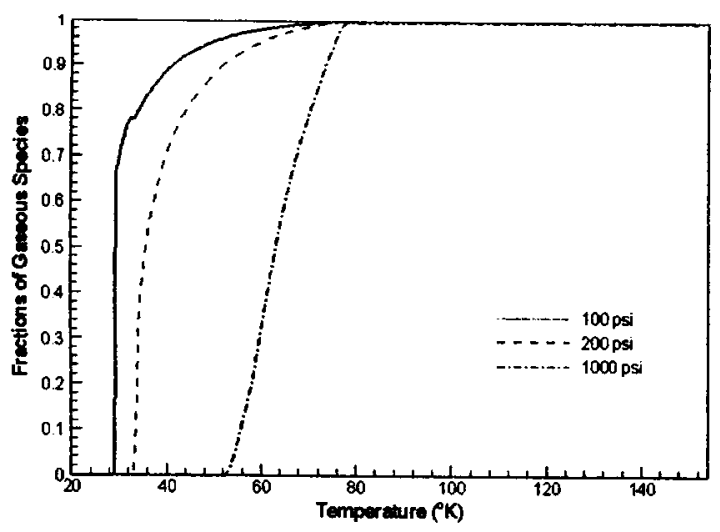

Figure 9. Fraction of gaseous hydrogen at various temperatures and pressures

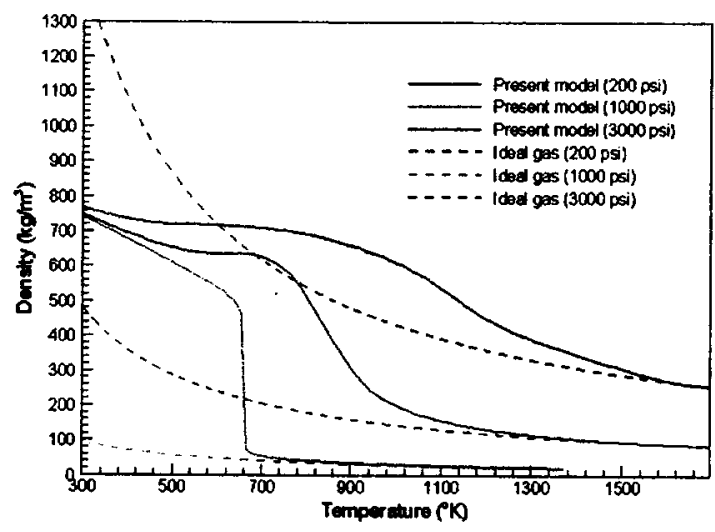

Figure 10. Comparison of RP-1 density at various temperatures and pressures

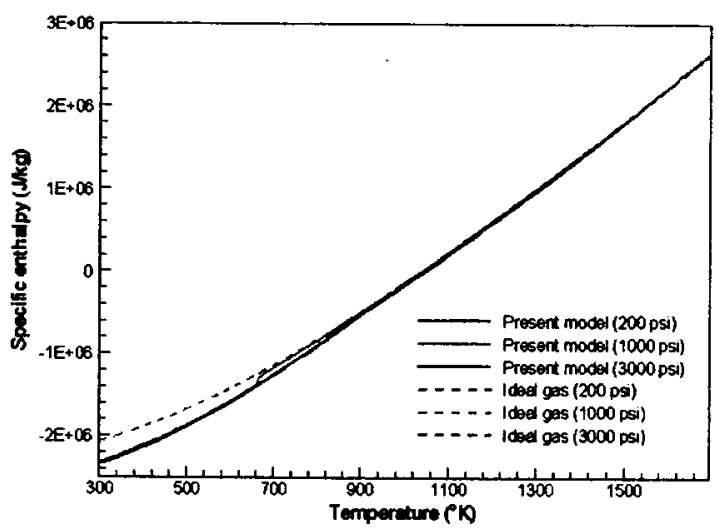

Figure 11. Comparison of RP-1 specific enthalpy at various temperatures and pressures

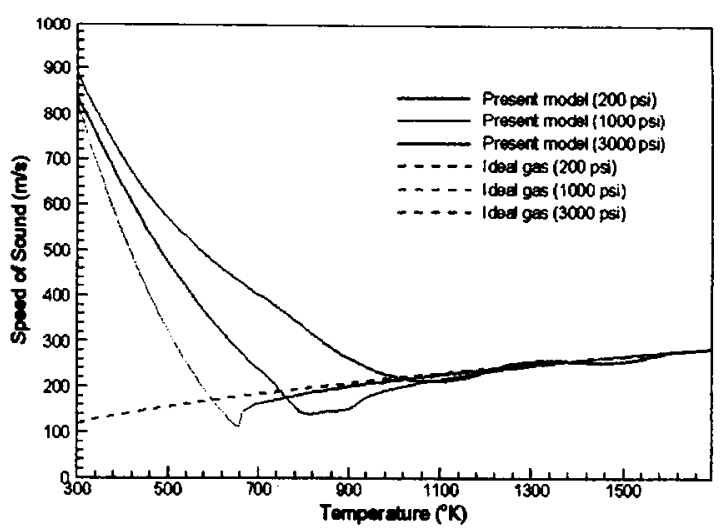

Figure 12. Comparison of RP-1 speed of sound at various temperatures and pressures 


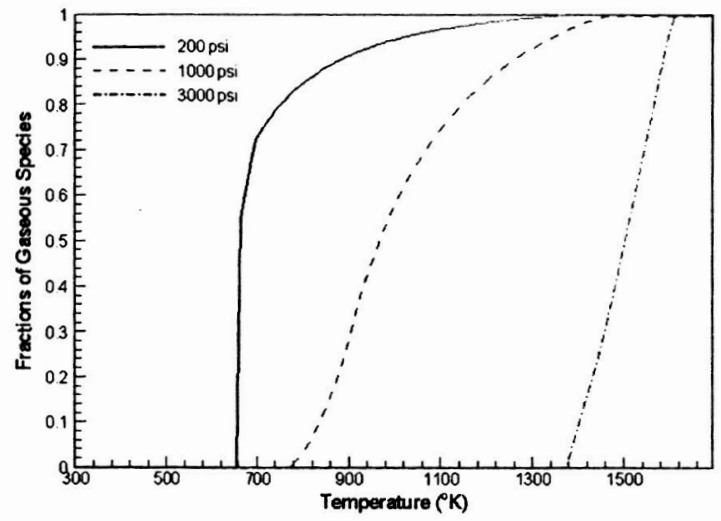

Figure 13. Fraction of gaseous RP-1 at various temperatures and pressures
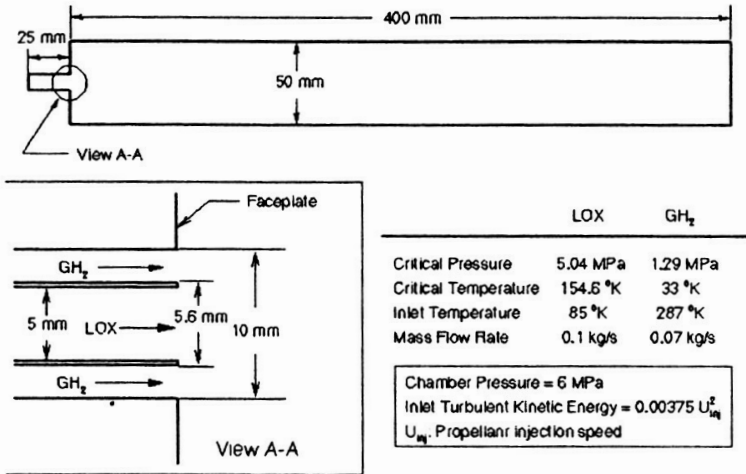

Figure 14. Configuration and flow conditions for the RCM-3 test case

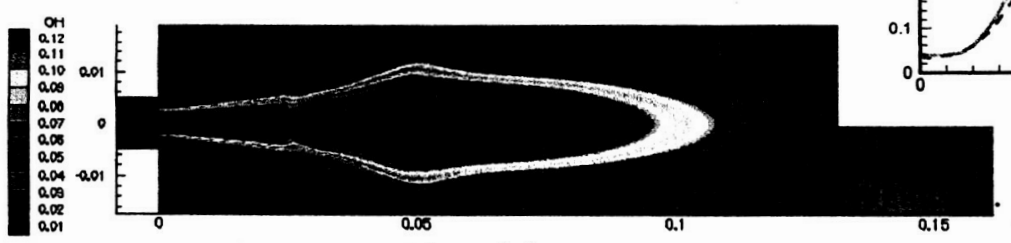

(a) Real-fluid model

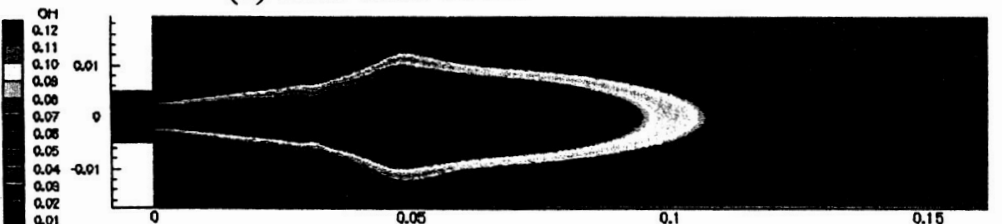

(b) Efficient real-fluid model

Figure 15. Comparisons of the $\mathrm{OH}$ concentrations near the injector face

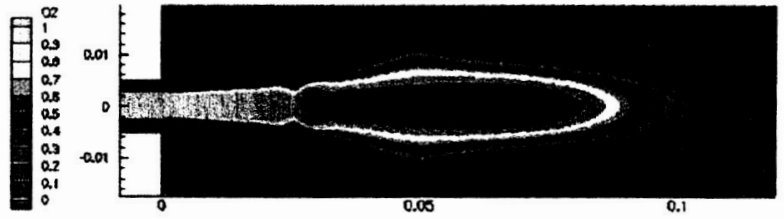

(a) Real-fluid model
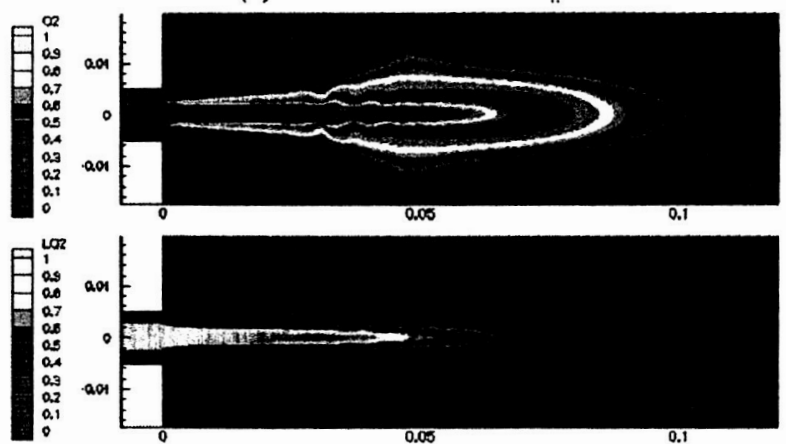

(b) Efficient real-fluid model

Figure 16. Comparisons of the oxygen concentrations near the injector face

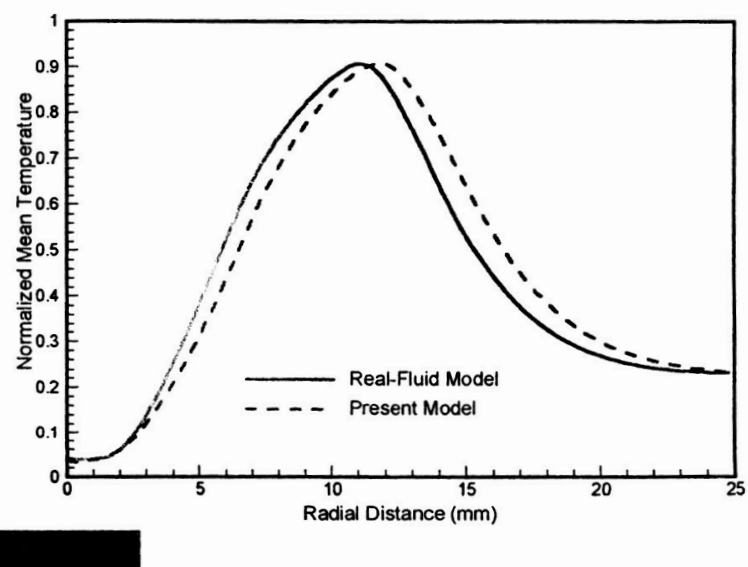

Comparisons of temperature profiles at $\mathrm{X} / \mathrm{D}=10(\mathrm{RCM}-3$ test case $)$ 


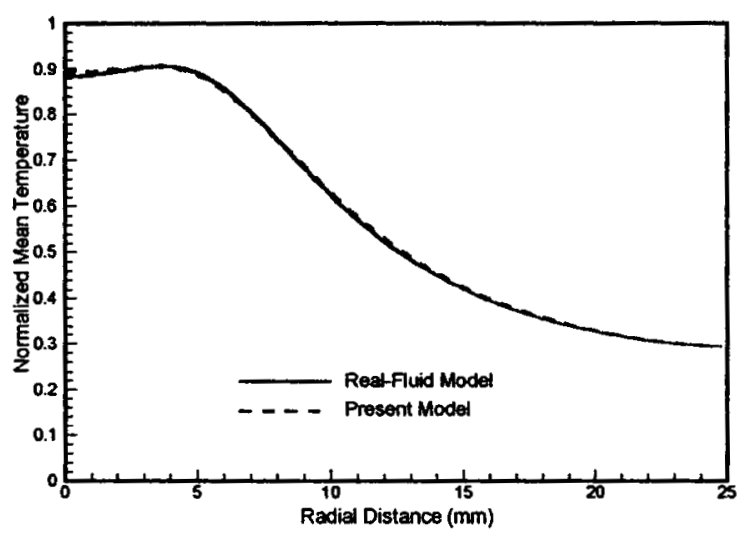

Figure 18. Comparisons of temperature profiles at $\mathrm{X} / \mathrm{D}=20$ (RCM-3 test case)

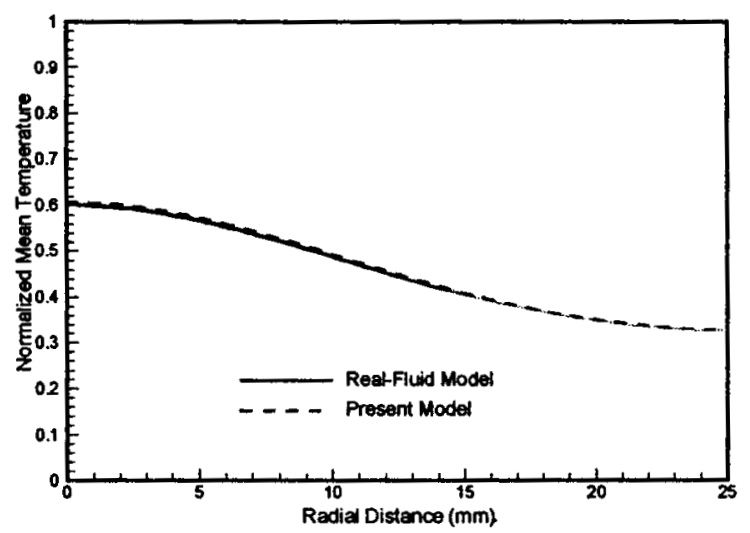

Figure 19. Comparisons of temperature profiles at $\mathrm{X} / \mathrm{D}=30(\mathrm{RCM}-3$ test case $)$

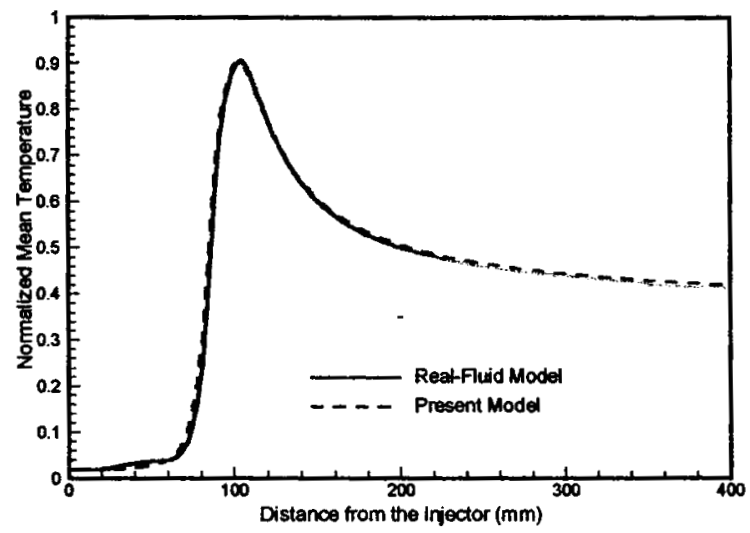

Figure 20. Comparisons of temperature profiles at $\mathrm{R} / \mathrm{D}=0$ (RCM-3 test case)

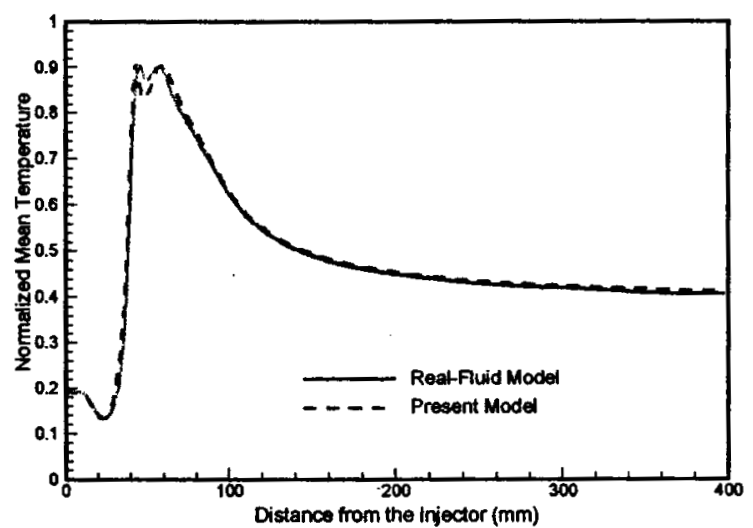

Figure 21. Comparisons of temperature profiles at $\mathrm{R} / \mathrm{D}=2$ (RCM-3 test case)

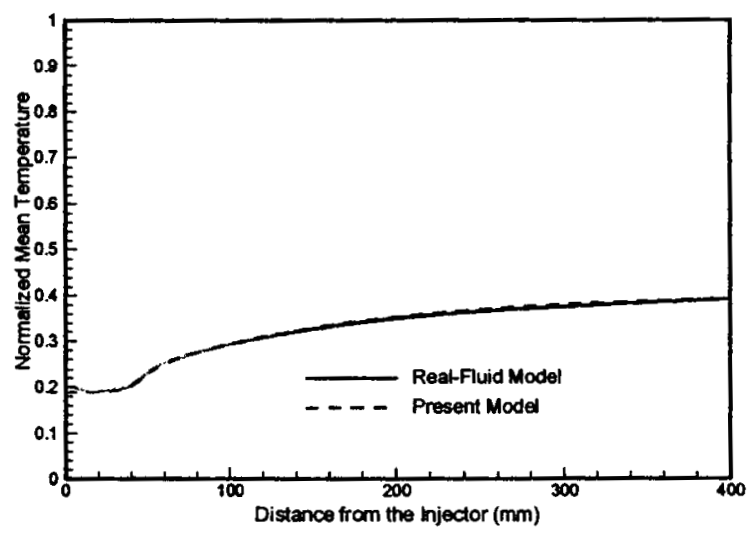

Figure 22. Comparisons of temperature profiles along the chamber wall (RCM-3 test case) 